\title{
LncRNA MONC Suppresses the Malignant Phenotype of Endometrial Cancer Stem Cells by Regulating the MiR-636/GLCE Axis
}

Yibing Li

Shengjing Hospital of China Medical University

Jianing Huo

Shengjing Hospital of China Medical University

Junjian He

Shengjing Hospital of China Medical University

Haining Ma

Shengjing Hospital of China Medical University

Xiaoxin Ma ( $\nabla$ maxiaoxin666@aliyun.com )

Shengjing Hospital of China Medical University

\section{Research}

Keywords: Endometrial carcinoma, endometrial cancer stem cells, LncRNA MONC, microRNA-636, GLCE, Notch signaling pathway, EMT

Posted Date: July 8th, 2020

DOl: https://doi.org/10.21203/rs.3.rs-40559/v1

License: (9) (1) This work is licensed under a Creative Commons Attribution 4.0 International License. Read Full License 


\section{Abstract}

Background: Emerging evidence shows that abnormal expression of long non-coding RNA is involved in the occurrence and development of various tumors. LncRNA MONC is abnormally expressed in head and neck squamous cell carcinoma (HNSCC), lung cancer, colorectal cancer, and acute megakaryocytic leukemia, but the biological function and potential regulatory mechanism of MONC in endometrial cancer stem cells (ECSCs) and endometrial cancer cells (ECCs) have not been studied. In this study, we aimed to explore the tumor suppressive effect and mechanism of MONC in regulating ECSCs and ECCs.

Methods: The expression of genes was detected by quantitative reverse transcription polymerase chain reaction (qRT-PCR). The expression of proteins was detected by Western blot. The interplay of LncRNAmiRNA-mRNA was verified using the luciferase assay. The growth rate of ECSC spheroids was detected by sphere formation assay. Cell proliferation was detected by CCK-8 assay. The cell invasion was detected by transwell invasion assay. Cell cycle was detected by Cell cycle analysis.Cell apoptosis was detected by the Annexin V-fluorescein isothiocyanate (FITC)/propidium iodide (PI) double-staining assay. Animal study was conducted to evaluate the effect of MONC combined with miR-636 on tumor growth in vivo.

Results: Low MONC expression in endometrial carcinoma (EC), which directly inhibits the malignant biological behavior of ECSCs and ECCs by directly inhibiting miR-636. Simultaneously, miR-636 may indirectly reduce the expression of MONC. Down-regulation of miR-636 may promote GLCE expression by targeting the 3'-untranslated region (UTR) of the downstream gene GLCE, thereby inhibiting the progression of ECSCs. MONC combined with miR-636 inhibited the Notch signaling pathway and tumor epithelial-to-mesenchymal transition (EMT) process. In addition, we verified the tumor suppressive effect of MONC in nude mice, miR-636 can rescue the tumor suppressive effect of overexpressing MONC, and this effect is more obvious in ECSC.

Conclusion: MONC inhibits the malignant phenotypes of ECSCs and ECCs by regulating the miR636/GLCE axis. The MONC/miR-636/GLCE axis may provide novel treatment avenues for human EC.

\section{Introduction}

Endometrial carcinoma (EC) is one of the three major malignant tumors in gynecology, with 61,880 new cases each year in the United States, second only to breast cancer, lung cancer, bronchial cancer, and colorectal cancer. There are 12,160 deaths each year, second only to lung cancer, bronchial cancer, breast cancer, colorectal cancer, pancreatic cancer, and ovarian cancer ${ }^{[1]}$. Risk factors for EC include elevated estrogen levels (caused by obesity, diabetes, and high-fat diets), premature menarche, non-parturients, late desperate age, Lynch syndrome, grade $\geq 55$ years old, and using tamoxifen [2].

Cancer stem cells (CSCs) are a class of cells that have the unique characteristics of self-renewal and the ability to differentiate into heterogeneous lineages of cancer cells ${ }^{[3,4]}$. CSCs can initiate tumor formation and promote tumor cell proliferation, while differentiation of component tumor cells plays a vital role in 
the occurrence, development, metastasis, recurrence, and drug resistance of malignant tumors ${ }^{[5,6]}$. In our previous research, we used serum-free suspension culture to isolate endometrial cancer stem cells (ECSCs) from Ishikawa cells ${ }^{[7]}$.

Long non-coding RNA (LncRNA) is a non-coding RNA with a length of more than 200 nucleotides. In recent years, LncRNA has been found to be an important biological marker for the diagnosis of tumors and their prognosis ${ }^{[8]}$. Increasing studies show that LncRNA plays a vital role in the occurrence and development of tumors, including EC, and LncRNA is dysregulated in EC and is closely related to tumorigenesis, metastasis, and chemoresistance ${ }^{[9]}$. However, LncRNA is rarely studied in ECSCs. MONC, mir-99a-let-7c cluster host gene, also known as MIR99AHG, is a good prognostic indicator of HNSCC [10], lung squamous cell carcinoma ${ }^{[11]}$, and colorectal cancer ${ }^{[12]}$. In acute megakaryoblastic leukemia, MONC acts as an oncogene to promote leukemia growth in AMKL cell lines and primary patient samples ${ }^{[13]}$. However, MIR99AHG has not been studied in EC and ECSCs.

MicroRNA (miRNA) is a non-coding RNA approximately 20-24 nucleotides in length, which can induce translational inhibition or degradation of target mRNA to inhibit gene expression [14]. Abnormally expressed miRNAs are associated with tumorigenesis, development, and response to treatment ${ }^{[15]}$. In recent years, miR-636 has been studied in tumors. Studies have shown that miR-636 is abnormally expressed in bladder cancer and liver cancer ${ }^{[16,17]}$. However, miR-636 has not been studied in EC and ECSCs.

In this study, we aimed to explore the expression of MONC in endometrial cancer tissues and ECSCs, and to clarify the interaction between MONC and miR-636 in ECSCs. It was found that miR-636 targeted MONC in a sequence-specific manner, suggesting that there may be mutual inhibition between miR-636 and MONC. To study the potential mechanism in this process, the effect of MONC on miR-636-induced GLCE regulation and its effect on the Notch signaling pathway and epithelial-mesenchymal transition (EMT) were also studied. Our findings shed light on new molecular mechanisms for the progression of EC and provide potential treatment options.

\section{Methods}

\section{Human tissue specimens}

All endometrial carcinoma tissue samples and normal endometrial tissue samples were obtained from patients undergoing total hysterectomy at Shengjing Hospital of China Medical University. The diagnosis of EC was evaluated by two experienced clinical pathologists based on FIGO for histological diagnosis and tumor grade. No patients received chemotherapy, radiotherapy, hormones, or treatment before surgery. All patients obtained informed consent and had been approved by the Ethics Committee of Shengjing Hospital, China Medical University (2018PS251K).

\section{ECSCs, cell lines, and cell culture}


ECSCs were cultured in serum-free medium, DMEM/F12 (1:1) (Corning, New York, USA) containing 2\% B27 Supplement (Gibco, New York, USA), 20 ng/ml EGF (PeproTech, New Jersey, USA), 20 ng/ml bFGF (PeproTech), and 1\% penicillin-streptomycin (Invitrogen, Carlsbad, California, USA). Ishikawa cell line (Shanghai huiying, Shanghai, China) and HEC-1A cell line (Genechem, Shanghai, China) were cultured in a-MEM medium (Bioind, Kibbutz Beit Haemek, Israel) and McCoy's 5A medium (Bioind), respectively. The medium contained 10\% fetal bovine serum (FBS) (Bioind) and 1\% penicillin-streptomycin (Invitrogen). HEK293T cells were cultured in DMEM/high-glucose medium (Corning). The medium contained 10\% FBS (Bioind) and 1\% penicillin-streptomycin (Invitrogen). All cells were cultured in a humidified incubator at 37 ${ }^{\circ} \mathrm{C}$ with $5 \% \mathrm{CO}_{2}$.

\section{RNA extraction, reverse transcription, and qRT-PCR}

Total RNA was extracted from tissues and cells using TRIzol reagent (Takara, Beijing, China). The complementary DNAs (cDNAs) for the IncRNAs and mRNAs of interest were reverse-transcribed from $2 \mu \mathrm{g}$ total RNA using PrimeScript RT-polymerase (Takara). The cDNAs for the miRNAs of interest were synthesized from $1 \mu \mathrm{g}$ total RNA using miRNA 1st Strand cDNA Synthesis SuperMix (Vazyme, Nanjing, China). We performed qRT-PCR using SYBR-Green Premix (Takara), miRNA Universal SYBR ${ }^{\circ}$ qPCR Master Mix (Vazyme), and specific PCR primers (Sangon Biotech, Shanghai, China). Glyceraldehyde-3phosphate dehydrogenase (GAPDH) and U6 were used as internal controls. We observed the expression of LncRNA, mRNA, and miRNA by calculating 2- $\triangle \triangle \mathrm{CT}$. Primer sequences are summarized in Table $\mathrm{S} 1$.

\section{Western blot}

Protein was extracted from tissues and cells using RIPA Lysis Buffer (Beyotime Biotechnology, Shanghai, China) and phenylmethanesulfonyl fluoride (PMSF) (Beyotime Biotechnology). Protein denaturation was performed after adding SDS-PAGE Sample Loading Buffer. Denatured proteins were separated by sodium dodecyl sulfate polyacrylamide gel electrophoresis (SDS-PAGE) and transferred to polyvinylidene fluoride membranes (Millipore, USA). Membranes were blocked with $5 \%$ skim milk for $2 \mathrm{~h}$ and washed thrice with TBST. Membranes were then incubated with diluted primary antibodies for GLCE (Abcom, Cambridge, United Kingdom), Notch1 (Proteintech, Chicago, USA), Notch-1 Intracellular Domain (N1ICD) (R\&D SYSTEMS, Minnesota, USA), Snail1 (Proteintech), E-cadherin (Proteintech), N-cadherin (Proteintech), and Vimentin (Proteintech) overnight at $4{ }^{\circ} \mathrm{C}$, and washed thrice with TBST thereafter. Membranes were then incubated with corresponding secondary antibodies for $2 \mathrm{~h}$, followed by washing thrice with TBST. The protein bands were visualized using BeyoECL Star (Beyotime Biotechnology) and Quantum One imaging software (Bio-Rad, California, USA), and normalized to the gray intensity of GAPDH.

\section{Transfection and generation of stably transfected cell line}

MONC lentiviral overexpression plasmid and lentiviral knockdown plasmid were purchased from GeneChem (Shanghai, China). ECSCs and Ishikawa cells were then transfected at a multiplicity of infection (MOI) of 20, while an $\mathrm{MOI}$ of 10 was used for HEC-1A cells. MiR-636 agomir and antagomir were purchased from GenePharma (Shanghai, China), and GLCE overexpression plasmid and knockdown 
plasmid were purchased from GeneChem (Shanghai). All cells were transfected with jetPRIME $\circledast$ in vitro DNA and siRNA Transfection Reagent (PolyPlus-transfection, France) for subsequent experiments. The relevant sequence can be found in Table S2.

\section{Flow cytometry sorting assay}

After Ishikawa cells were cultured and passaged in serum-free medium, the cell pellet was collected and washed once with phosphate-buffered saline (PBS). Cells were resuspended (107 cells in $100 \mu \mathrm{lPBS}$ ) and labeled with APC Mouse Anti-Human CD133 (BD Biosciences, New Jersey, USA) and PerCP-Cy7M 5.5 Mouse Anti-Human CD44 (BD Biosciences). Following incubation for 10 minutes in the dark, cells were washed with PBS, and the supernatant was discarded after centrifugation. Thereafter, cells were resuspended in PBS, and flow sorted using the BD FACSAriaTM III Cell Sorter (BD Biosciences).

\section{Sphere formation assay}

After obtaining ECSCs in the above manner, cells were cultured in DMEM/F12 (1:1) serum-free medium containing $2 \%$ B27 additive, $20 \mathrm{ng} / \mathrm{ml} \mathrm{EGF,} 20 \mathrm{ng} / \mathrm{ml} \mathrm{bFGF}$, and 1\% penicillin-streptomycin After culture for 3-5 days, 5000 cells were seeded in ultra-low-attachment 6-well plates (Corning, USA) and cultured for 7 days further. Cells were imaged under an inverted fluorescence microscope and an image acquisition system. The sphere diameter was measured at the time of inoculation and 7 days, respectively.

\section{CCK-8 assay}

Cells were cultured in 96-well plates (Guangzhou Jet Bio-Filtration Co., Ltd.), CCK-8 reagent (10 $\mu$ l) (Dojindo, Japan) was added to each well, and then incubated at $37{ }^{\circ} \mathrm{C}$ with $5 \% \mathrm{CO} 2$ for 3 hours. The OD450 value of each well was determined using a microplate reader. Detection was performed at $12 \mathrm{~h}, 24$ $\mathrm{h}, 48 \mathrm{~h}$, and $72 \mathrm{~h}$ after treatment.

\section{Transwell invasion assay}

Transwell filter inserts ( $8 \mu \mathrm{m}$ pore size; Corning) were pre-coated with Matrigel at $37^{\circ} \mathrm{C}$ for 30 minutes. Complete medium $(500 \mu \mathrm{l})$ was added to each well of the 24-well plate, and pure medium cell suspension $(200 \mu \mathrm{l})$ was added to the chamber. After incubation for 24 hours, cells were fixed with $4 \%$ polyoxymethylene for 30 minutes, then stained with $0.1 \%$ crystal violet for 30 minutes. Cells were imaged under an inverted fluorescence microscope and an image acquisition system (Nikon, Japan).

\section{Cell cycle analysis}

After cell transfection, 106 cells were collected from each group. After washing once in PBS, cells were fixed in $70 \%$ ethanol at $4{ }^{\circ} \mathrm{C}$ overnight. After washing once in PBS, cells were resuspended in $500 \mu \mathrm{l}$ PBS, followed by the addition of $10 \mu \mathrm{l}$ RNase $A$ and $5 \mu$ propidium iodide, then incubated at $37^{\circ} \mathrm{C}$ for 30 minutes. Flow cytometry (BD FACSCalibur, New Jersey, USA) was used to evaluate the proportion of cells at different stages of the cell cycle. 
After cell transfection, 106 cells were collected from each group. After washing once in PBS, cells were stained with PE Annexin $V$ and 7AAD using PE Annexin V Apoptosis Detection (BD Pharmingen ${ }^{\mathrm{TM}}$, New Jersey, USA) at room temperature for 15 minutes. We used flow cytometry (BD FACSCalibur, New Jersey, USA) to evaluate the proportion of apoptotic cells.

\section{Luciferase assay}

The bioinformatics website, RNAhybrid, was used to predict the potential binding sites between MONC and miR-636. MONC wild-type and mutant dual-luciferase reporter vectors were purchased from Liaoning Baihaobio Biotech Co., Ltd. (Liaoning, China), and co-transfected with miR-636 agomir or NC (GenePharma, Shanghai). The bioinformatics website, miRDB, was used to predict the potential binding sites between miR-636 and GLCE. GLCE wild-type and mutant dual-luciferase reporter vectors were purchased from Liaoning Baihaobio Biotech Co., Ltd. (Liaoning, China), and co-transfected with miR-636 agomir or NC. A dual-luciferase reporter gene detection system (Promega, Madison, WI, USA) was used to detect luciferase activity.

\section{Tumor xenografts in nude mice}

Transfected cells were evaluated in vivo in nude mice. We conducted experiments in strict accordance with a protocol approved by the Administrative Panel on Laboratory Animal Care of the Shengjing Hospital (2018PS136K). Nude mice were purchased from HFK Bioscience (Beijing, China), and all were 4week-old BALB/C athymic nude mice. Each mouse was injected with $5 \times 106$ cells at the armpit. The tumor volume was calculated according to the following formula: tumor volume $(\mathrm{mm} 3)=$ length $\times$ width $2 / 2$. The experiment was conducted in compliance with the Institutional Animal Care and Use Committee standards. When the mice developed tumor metastasis, lethargy, weight loss $\geq 20 \%$, or other signs of discomfort that met the IACUC criteria, the mice were sacrificed by cervical dislocation.

\section{Statistical analysis}

Data are expressed as Mean \pm Standard Error of Mean (SEM). All statistical analyses were performed using GraphPad Prism 8.0 Software (La Jolla, CA, USA) and SPSS version 22.0 software (Abbott Laboratories, Chicago, IL, USA) through two-sided Student's t-test or one-way analysis of variance (ANOVA). Differences were considered statistically significant at $P<0.05$.

\section{Results:}

\section{MONC exhibits low expression in EC as a tumor suppressor gene}


By quantitative real-time polymerase chain reaction (qRT-PCR) detection, compared with normal human endometrial tissue, MONC exhibited low expression in human endometrial carcinoma tissue (Fig. 1A). Clinical pathological analysis showed that MONC expression was related to the depth of invasion and FIGO Stage (Table 1). To determine the effect of MONC on ECSCs, we used LV-MONC (+), LV-MONC-RNAi $(-)$, and their corresponding negative controls to construct stable lentiviral ECSCs, and Ishikawa and HEC$1 \mathrm{~A}$ cell lines. Transfection efficiency was verified using qRT-PCR. A lentiviral vector (LV-MONC-RNAi (67379-1)) with the best knockdown effect was selected for subsequent experiments (Figure S1A).

Table 1

Relationship between MONC expression and pathologic tumor parameters

\begin{tabular}{|lrll|}
\hline Clinical parameters & $\mathbf{n}$ & The expression of MONC (Mean \pm SEM) & $\mathbf{P}$ \\
\hline Differentiation & & & 0.098 \\
\hline High + High-Middle & 35 & $0.360 \pm 0.036$ & \\
\hline Middle + Middle-Low + Low & 25 & $0.467 \pm 0.056$ & 0.002 * \\
\hline Invasion depth & & & \\
घ1/2 & 40 & $0.473 \pm 0.041$ & 0.007 * \\
$\geq 1 / 2$ & 20 & $0.268 \pm 0.035$ & \\
\hline FIGO Stage & & & 0.241 \\
\hline I & 52 & $0.438 \pm 0.032$ & \\
\hline II + III + IV & 8 & $0.189 \pm 0.088$ & \\
\hline Age, y & & & \\
\hline$\square 60$ & 34 & $0.438 \pm 0.046$ & \\
\hline$\geq 60$ & 26 & $0.362 \pm 0.042$ & \\
\hline
\end{tabular}

Based on previous research by our team, in order to extract ECSCs from endometrial carcinoma cells (ECCs), we used DMEM/F12 (1:1) serum-free medium containing 2\% B27 supplement, $20 \mathrm{ng} / \mathrm{ml}$ EGF, $20 \mathrm{ng} / \mathrm{ml} \mathrm{bFGF}$, and 1\% penicillin-streptomycin for Ishikawa cells to form mammospheres. Post-sphere formation, Ishikawa cells were labeled with CD44 (PerCP-Cy7M5.5 Mouse Anti-Human) and CD133 (APC Mouse Anti-Human), cells positive for both CD44 and CD133 were obtained using flow cytometry cell sorting. We obtained ECSCs via flow sorting, and these were used in subsequent experiments (Figure S1CD).

In the CCK-8 cell proliferation assay, we observed reduced proliferation rates in the MONC overexpression group, while the MONC knocked down group appeared increased (Fig. 1B). In the ECSC sphere formation assay, we found that the growth rate of ECSC spheroids in the MONC overexpression group slowed down, while the growth rate of ECSC spheroids in the MONC knockdown group accelerated (Fig. 1C). Thereafter, 
the Transwell cell invasion assay revealed that overexpression of MONC inhibited the invasion of ECSCs, and Ishikawa and HEC-1A cells. Conversely, knockdown of MONC promoted the invasion of ECSCs, and Ishikawa and HEC-1A cells (Fig. 1D). Next, we examined the effects on overexpression and knockdown of MONC on the cell cycle and apoptosis of ECSCs, and Ishikawa and HEC-1A cells using flow cytometry. The results showed that MONC overexpression induced cell cycle arrest in the G0/G1 phase, while knockdown of MONC exerts the opposite effect (Fig. 1E). Overexpression of MONC promotes apoptosis, and knockdown of MONC inhibits apoptosis (Fig. 1F).

Therefore, MONC exhibits low expression in endometrial carcinoma as a tumor suppressor gene.

\section{Mir-636 exhibits high expression in EC as an oncogene, while MONC binds and negatively regulates miR- 636}

qRT-PCR revealed that miR-636 was highly expressed in human endometrial carcinoma tissue compared with normal human endometrial tissue (Fig. 2A). Clinical pathological analysis showed that the expression of MONC is related to invasion depth and FIGO Stage (Table 2). In addition, the expression of miR-636 was negatively correlated with the expression of MONC (Fig. 2B, Pearson's rank correlation method: $r 2=0.0945, P=0.0169$ ).

Table 2

Relationship between miR-636 expression and pathologic tumor parameters.

\begin{tabular}{|c|c|c|c|}
\hline Clinical parameters & $\mathrm{n}$ & The expression of miR-636 (Mean \pm SEM) & $P$ \\
\hline Differentiation & & & 0.339 \\
\hline High + High-Middle & 35 & $6.400 \pm 0.779$ & \\
\hline Middle + Middle-Low + Low & 25 & $7.525 \pm 0.844$ & \\
\hline Invasion depth & & & 0.037 * \\
\hline$\nabla 1 / 2$ & 40 & $6.027 \pm 0.601$ & \\
\hline$\geq 1 / 2$ & 20 & $8.552 \pm 1.167$ & \\
\hline FIGO Stage & & & $\triangle 0.001$ * \\
\hline 1 & 52 & $6.039 \pm 0.526$ & \\
\hline$I I+I I I+I V$ & 8 & $12.260 \pm 1.710$ & \\
\hline Age, y & & & 0.987 \\
\hline$\nabla 60$ & 34 & $6.877 \pm 0.818$ & \\
\hline$\geq 60$ & 26 & $6.858 \pm 0.800$ & \\
\hline
\end{tabular}

A bioinformatics database (RNAhybrid) was used to predict the binding site between MONC and miR-636. In order to further study whether MONC is a functional target of miR-636, we co-transfected HEK-293T 
cells with MONC-WT and NC, and MONC-WT and miR-636. Thereafter, we performed a dual-luciferase reporter assay. The results showed that the relative luciferase activity of the MONC-WT + miR-636 group was lower than that of the MONC-WT + NC group, suggesting that there is a binding site between MONC and miR-636 (Fig. 2E). A MONC mutant vector was constructed, based on the predicted binding sites of the bioinformatics database (RNAhybrid). Next, we co-transfected HEK-293T cells with MONC-Mut and NC, and MONC-Mut and miR-636. Detection of the dual-luciferase reporter assay verified the binding site between MONC and miR-636 (Fig. 2E).

We used qRT-PCR to determine the expression of miR-636 in ECSCs, Ishikawa cells, and HEC-1A cells stably transfected with MONC overexpression and knockdown. The results showed that the expression of miR-636 decreased in the MONC overexpression group, while miR-636 expression increased in the MONC knockdown group (Fig. 2C). Next, we transfected ECSCs, Ishikawa cells, and HEC-1A cells with AgomiR636, miR-636 antagomir, and corresponding negative controls. We detected the expression of MONC using qRT-PCR. The results showed that MONC expression decreased in the AgomiR-636 group and increased in the miR-636 antagomir group (Fig. 2D).

Therefore, we speculate that miR-636 is overexpressed in EC as an oncogene, MONC binds and negatively regulates miR-636, and there may be a feedback loop of mutual inhibition between MONC and miR-636.

\section{Knockdown of miR-636 mediates the tumor suppressive effect of MONC overexpression in ECSCs and ECCs}

To determine whether miR-636 mediated tumor suppression via MONC overexpression, we first transfected miR-636 antagomir into ECSCs, Ishikawa cells, and HEC-1A cells stably overexpressing MONC. AgomiR-636 was transfected into MONC knocked down ECSCs, Ishikawa cells, and HEC-1A cells. We divided the experiments into six groups: Control, MONC (+), miR-636 (-), Stable NC, MONC(+) + miR636(-), and MONC(-) + miR-636(+).

The CCK-8 cell proliferation assay indicated that the MONC(+) + miR-636(-) group exhibited the lowest proliferation rate, while the MONC(-) + miR-636(+) group exhibited the highest proliferation rate (Fig. 3A). The sphere formation assay demonstrated that miR-636 knockdown can inhibit the growth rate of ECSC spheroids. The MONC(+) + miR-636(-) group displayed the most obvious inhibitory effect on the growth rate of ECSC spheroids. The ECSC spheroids in the MONC(-) + miR-636(+) group displayed the fastest growth rate (Fig. 3B). According to the Transwell cell invasion experiment, we found that knockdown of miR-636 inhibited the invasion of ECSCs, Ishikawa cells, and HEC-1A cells. The MONC(+) + miR-636(-) group displayed the strongest invasion inhibitory effects in ECSCs, Ishikawa cells, and HEC-1A cells, while the MONC(-) + miR-636(+) group displayed the weakest invasion inhibitory effects (Fig. 3C). Thereafter, we employed flow cytometry to observe the cell cycle and apoptosis of ECSCs, Ishikawa cells, and HEC$1 \mathrm{~A}$ cells after transfection. The results showed that AntagomiR-636 can induce cell cycle arrest at the G0/G1 phase, the MONC (+) + miR-636(-) group had the strongest ability to induce cell cycle arrest in the G0/G1 phase, and the MONC(-) + miR-636(+) group had the weakest ability (Fig. 3D). MiR-636 can inhibit 
apoptosis, and the MONC(+) + miR-636(-) group exhibited the strongest inhibitory effect on apoptosis, while the MONC(-) + miR-636(+) group exhibited the weakest (Fig. 3E).

Therefore, we believe that miR-636 can mediate tumor suppressive effects of MONC overexpression in ECSCs, Ishikawa cells, and HEC-1A cells, and that knockdown of MONC combined with overexpression of miR-636 have significant carcinoma-promoting effects in ECSCs and ECCs.

\section{GLCE exhibits low expression in EC as a tumor suppressor gene, and participates in the malignant progression of ECSCs and ECCs induced by MONC-miR-636}

qRT-PCR and western blot experiments revealed low expression of GLCE in human endometrial carcinoma tissue compared to normal human endometrial tissue (Fig. 4A-B). Clinical pathological analysis showed that the expression of GLCE is related to differentiation, invasion depth, and FIGO Stage (Table 3).

Table 3

Relationship between GLCE expression and pathologic tumor parameters.

\begin{tabular}{|c|c|c|c|}
\hline Clinical parameters & $\mathbf{n}$ & The expression of GLCE (Mean \pm SEM) & $\mathbf{P}$ \\
\hline Differentiation & & & 0.003 * \\
\hline High + High-Middle & 35 & $0.193 \pm 0.033$ & \\
\hline Middle + Middle-Low + Low & 25 & $0.371 \pm 0.050$ & \\
\hline Invasion depth & & & 0.023 * \\
\hline$\otimes 1 / 2$ & 40 & $0.316 \pm 0.039$ & \\
\hline$\geq 1 / 2$ & 20 & $0.170 \pm 0.042$ & \\
\hline FIGO Stage & & & 0.012 * \\
\hline I & 52 & $0.297 \pm 0.033$ & \\
\hline$I I+I I I+I V$ & 8 & $0.076 \pm 0.021$ & \\
\hline Age, y & & & 0.104 \\
\hline$\nabla 60$ & 34 & $0.311 \pm 0.042$ & \\
\hline$\geq 60$ & 26 & $0.210 \pm 0.043$ & \\
\hline
\end{tabular}

Using the bioinformatics database (miRDB), we observed that GLCE is a downstream target gene of miR636. Therefore, we co-transfected HEK-293T cells with GLCE-WT and NC, and GLCE-WT and miR-636. We conducted dual-luciferase reporter tests and discovered that the relative luciferase activity of the GLCEWT + miR-636 group was lower than that of the GLCE-WT + NC group, suggesting that there is a binding site between GLCE and miR-636 (Fig. 4C). A GLCE mutant vector was constructed based on the predicted binding sites of the bioinformatics database (miRDB), followed by co-transfection of GLCE-Mut and NC, 
and GLCE-Mut and miR-636. The dual-luciferase reporter test verified the binding site between MONC and miR-636 (Fig. 4C).

In order to investigate whether MONC could regulate miR-636 in EC as ceRNA of GLCE, western blot was used to determine the expression level of GLCE in ECSCs, Ishikawa cells, and HEC-1A cells transfected with MONC. MONC overexpression promotes expression of GLCE at the protein level. Simultaneously, transfection with AgomiR-636 rescued MONC from promoting GLCE protein expression (Fig. 4D).

To explore the effect of GLCE on the malignant biological behavior of ECSCs, we first transfected ECSCs, Ishikawa cells, and HEC-1A cells with GLCE overexpression plasmids, GLCE knockdown plasmids, and corresponding negative controls. The transfection efficiency was verified using qRT-PCR. A plasmid with the best knockdown effect (GLCE-RNAi(5952-1)) was selected for subsequent experiments (Figure S1B). Next, we performed a CCK-8 cell proliferation experiment and found that the proliferation rate in the GLCEoverexpressed group decreased, while the proliferation rate in the GLCE-knocked-down group increased (Fig. 5A). The sphere formation assay indicated that the growth rate of ECSC spheroids in the GLCE overexpression group slowed down, while that in the GLCE knockdown group accelerated (Fig. 5B). We observed that the overexpression of GLCE inhibited the invasion of ECSCs, Ishikawa cells, and HEC-1A cells. Conversely, knockdown of GLCE promoted the invasion of ECSCs, Ishikawa cells, and HEC-1A cells (Fig. 5C). Next, we used flow cytometry to examine the effects of GLCE knockdown and overexpression on the cell cycle and apoptosis of ECSCs, Ishikawa cells, and HEC-1A cells. The results showed that overexpression of GLCE can induce cell cycle arrest at the G0/G1 phase, while knockdown of GLCE exerts the opposite effect (Fig. 5D). Overexpression of GLCE promotes apoptosis, and GLCE knockdown inhibits apoptosis (Fig. 5E).

Therefore, we speculated that GLCE expression is low in EC as a tumor suppressor gene and participates in the malignant progression of ECSCs and ECCs induced by MONC-miR-636. Among them, MONC can regulate miR-636 as the ceRNA of GLCE.

\section{Overexpression of MONC inhibits the Notch signaling pathway and inhibits the EMT process in ECSCs and ECCs, while miR-636 rescues the inhibitory effect of MONC overexpression.}

In order to explore the molecular mechanism of the downstream signaling pathway regulated by MONC and miR-636, we first constructed ECSCs, and Ishikawa and HEC-1A cell lines stably overexpressing MONC. AgomiR-636 was transfected into ECSCs, Ishikawa cells, and HEC-1A cells stably overexpressing MONC. We divided the experiment into three groups, namely, Control, MONC(+), and MONC $(+)+$ miR$636(+)$. The protein levels of the Notch signaling pathway and EMT-related indicators were detected using western blot. The results showed that MONC overexpression inhibited the expression of Notch1 and N1 ICD protein levels, inhibited the expression of Snail1, Vimentin, and N-cadherin, and promoted the expression of E-cadherin. miR-636 can rescue the inhibitory effects of MONC overexpression on the Notch1 signaling pathway and EMT process (Fig. 6A). 
Therefore, we speculate that the knockdown of MONC overexpression in combination with miR-636 inhibits the Notch signaling pathway and the EMT process in ECSCs and ECCs.

\section{Overexpression of MONC inhibited the growth of EC tumors in nude mice. miR-636 rescued the inhibitory effect of MONC overexpression on the growth of EC tumors}

We conducted nude mice tumorigenesis experiments to investigate the effect of MONC combined with miR-636 on tumor growth in nude mice. MONC(+) and MONC(+) + miR-636(+) ECSCs, Ishikawa cells, and HEC-1A cells were injected subcutaneously into nude mice.

The results are shown in Fig. 7. In the MONC(+) group and the MONC(+) + miR-636(+) group, the tumor volume is smaller than that of the control group, the MONC(+) group exhibits the smallest tumor volume, and this effect is more obvious in ECSC (Fig. 7A). Further, we detected the expression of GLCE in the tumor using western blot. The expression of GLCE in the tumors of the MONC(+) group and the MONC(+) + miR-636(+) group increased, while GLCE expression is highest in the MONC(+) group (Fig. 7B). Results indicate that MONC can inhibit the growth of EC tumors, and miR-636 rescued MONC from inhibiting the growth of EC tumors.

\section{Discussion}

Endometrial carcinoma (EC) is one of the three major malignant tumors of the female reproductive system ${ }^{[1]}$. The risk factors are related to age, obesity, hypertension, excessive endometrial exposure to estrogen, diabetes, and hereditary nonpolyposis colorectal cancer. At present, the main treatment is still total hysterectomy with bilateral salpingo-oophorectomy, while radiation and chemotherapy can also play therapeutic roles ${ }^{[18]}$.

CSCs can promote tumor growth, recurrence, metastasis, and drug resistance ${ }^{[19]}$. The functional role of CSCs in the generation and recurrence of malignant tumors has two key characteristics: the self-renewal capacity of CSCs and the potential to differentiate into an infinite group of heterogeneous cancer cells ${ }^{[20]}$. Due to the plasticity of CSCs, Stationary CSCs may produce cycling CSCs, leading to cancer recurrence ${ }^{[21]}$. Studies have shown that PKA activation promotes CSC state in small cell lung cancer ${ }^{[22]}$. In colorectal cancer, CSC evades treatment-mediated DNA damage by changing cell cycle checkpoints, increasing DNA damage repair capabilities, and effectively removing reactive oxygen species ${ }^{[23]}$. In recent years, there have been reports of ECSCs, but there are few related studies. Studies have found selective and specific effects of metformin on the activity of ECSCs ${ }^{[24]}$. In another study, SPIONs were found to be highly efficient nanocarriers for nucleic acids. Loading miR-326 on these carriers inhibited the activation of the GPR91/STAT3/VEGF signaling pathway and significantly reduced ECSC activity [25]. Moreover, our group used serum-free suspension culture to isolate ECSCs from Ishikawa cells in previous research ${ }^{[6]}$. In this study, we used serum-free suspension culture to isolate ECSCs from Ishikawa cells for subsequent experiments. 
The mechanism of action of LncRNA in different tumors is inconsistent, and includes changes in histone modification, regulation of classic stem cell-related signaling pathways (such as SOX2/KLF4), induction of EMT, and inhibition of miRNA function ${ }^{[26]}$. Abnormal expression of LncRNA plays a key role in selfrenewal, effective transformation of CSCs, and tumor progression. For example, the well-known LncRNA HOTAIR is significantly up-regulated and promotes CSC properties in breast and colon CSCs ${ }^{27,28]}$. LncRNA HAND2-AS1 promotes self-renewal of liver CSCs and drives liver cancer ${ }^{[29]}$. LncRNA DLX6-AS1 down-regulation may inhibit methylation of the CADM1 promoter and the inactivation of the STAT3 signaling pathway, and up-regulate CADM1 to suppress stem cell characteristics of liver CSCs ${ }^{[30]}$. In related research on EC, Linc-RNA-RoR acts as a "sponge", preventing microRNA-145 from mediating ECSC differentiation ${ }^{[31]}$.

LncRNA can show similar effects as tumor suppressor genes or oncogenes by affecting various cellular processes related to cancer, including cell growth, metastasis, differentiation, and stemness ${ }^{[32]}$. LncRNA can act as competitive endogenous RNA (ceRNA) or a natural microRNA sponge, communicate with each other, and regulate together through the combination of competition and shared microRNA ${ }^{[33]}$. For example, LncRNA HOTTIP facilitates the development of breast cancer by regulating the miR-148a3p/WNT1 pathway ${ }^{[34]}$. LncRNA ZEB2-AS1, through the miR-574-3p/HMGA2 axis, promotes the proliferation, migration, and invasion of esophageal squamous cell carcinoma cells ${ }^{[35]}$. Previous research indicates that LncRNA PVT1 promotes malignant tumors of human ECCs through the negative regulation of miR-195-5p [36] and LINC01016 promotes the malignant phenotype of ECCs by regulating the miR-302a-3p/miR-3130-3p/NFYA/SATB1 axis ${ }^{[37]}$. In this article, we found that LncRNA MONC is lowly expressed in EC. MONC up-regulation inhibits the growth rate of ECSC spheres, while inhibiting proliferation and invasion, promoting apoptosis, and inducing cell cycle arrest at the G0/G1 phase in ECSCs, Ishikawa cells, and HEC-1A cells. Next, in order to evaluate the function of MONC, we predicted the binding site between MONC and miR-636 using RNAhybrid and verified the binding site between them using dual luciferase reporter gene detection. In addition, miR-636 knockdown can mediate tumor suppression related to MONC overexpression in ECSCs. In order to further explore the regulatory mechanism of MONC, we found that GLCE, as a tumor suppressor gene, is lowly expressed in EC and is the target mRNA of miR-636. MONC up-regulation promoted GLCE protein expression, while miR-636 rescued the promotion effect of MONC up-regulation on GLCE. Functional experiments show that GLCE up-regulation inhibits the growth rate of ECSC spheres, while also inhibiting proliferation and invasion, promoting apoptosis, and inducing cell cycle arrest at the G0/ G1 phase in ECSCs, Ishikawa cells, and HEC-1 A cells. We believe that the MONC/miR-636/GLCE axis plays a crucial role in sphere formation, proliferation, invasion, apoptosis, and induction of cell cycle arrest in ECSCs and ECCs. This information could provide future treatment options for EC.

The Notch signaling pathway is an evolutionarily conserved pathway that is closely related to all aspects of cancer biology, including CSC process, angiogenesis, and tumor immunity ${ }^{[38]}$. Activation of the Notch signaling pathway promotes tumor proliferation and metastasis. Conversely, inhibition of this pathway 
can eliminate CSCs and increase drug sensitivity ${ }^{[39]}$. For example, Notch signaling is a key method to regulate the survival of CSCs in triple negative breast cancer. Treatment of triple-negative breast cancer with PI3K or mTORC1/2 inhibitors will produce drug-resistant Notch-dependent CSCs ${ }^{[40]}$. Most tumors are affected by EMT, the process of obtaining mesenchymal features from epithelial cells, during tumor progression. It is generally believed that cancers derived from epithelia are determined by the EMT process ${ }^{[41]}$. In human malignant tumors, among the transcription factors involved in EMT, Snail plays a major inducing role, while Twist and Zeb1/2 are mainly involved in retaining the aggressive mesenchymal phenotype. Activation of EMT is related to the generation of CSCs, and a connection exists between EMT, stemness, and the metastatic initiation potential of tumor cells ${ }^{[42]}$. Further, the Notch signaling pathway is related to the EMT process. Studies have shown that LincRNA-ROR is activated by H3K27 acetylation and activates the Notch signaling pathway by acting as a miR-32 sponge, thereby inducing EMT in retinoblastoma ${ }^{[43]}$. In pancreatic cancer cells, Notch signaling is involved in the acquisition of EMT and cancer stem-like phenotypes ${ }^{[44]}$. In breast cancer, Notch signaling regulates EMT, invasion, and growth of breast cancer cells by inducing Slug expression ${ }^{[45]}$. In squamous cell carcinoma, the combination of Notch 1 activation and EMT promotes the presence of transforming growth factor (TGF) $\beta$ in the tumor microenvironment to promote tumor development ${ }^{[46]}$. The induction of EMT and CSC properties is also related to lung cancer risk of PM2.5 in the body. Blocking Notch1 may produce a negative regulatory effect on EMT and CSCs, thereby inhibiting invasion and migration in vitro, and it is speculated that it may play a role in the body ${ }^{[47]}$. In this article, MONC overexpression inhibits the expression of Notch1 and N1ICD protein levels, inhibits the expression of Snail1, Vimentin, and Ncadherin, and promotes the expression of E-cadherin. miR-636 can rescue the inhibitory effect of MONC overexpression on the Notch signaling pathway and EMT process. We speculate that in ECSCs, MONC combined with miR-636 inhibits the Notch signaling pathway and tumor EMT process.

In this study, we believe that the MONC/miR-636/GLCE axis has an important effect on the malignant phenotype of endometrial cancer stem cells. However, whether this axis can be used for the treatment of endometrial cancer requires more research.

\section{Conclusions}

Our study confirmed, for the first time, that MONC inhibits the malignant biological behavior of ECSCs and ECCs by directly inhibiting miR-636. In addition, miR-636 may indirectly reduce the expression of MONC. Down-regulation of miR-636 may promote the expression of GLCE by targeting the 3 '-untranslated region (UTR) of the downstream gene GLCE, thereby inhibiting the progression of ECSCs and ECCs. MONC combined with miR-636 inhibited the Notch signaling pathway and tumor EMT process. In addition, we conducted animal studies to confirm the effect of the combination of MONC and miR-636 on tumor growth in vivo. We speculate that the MONC/miR-636/GLCE axis may provide a new therapeutic strategy for the treatment of human EC.

\section{Abbreviations}


ECSCS

endometrial cancer stem cells

ECCs

endometrial cancer cells

EC

endometrial carcinoma

UTR

untranslated region

EMT

epithelial-to-mesenchymal transition

CSCs

Cancer stem cells

LncRNA

Long non-coding RNA

miRNA

MicroRNA

FBS

fetal bovine serum

PMSF

phenylmethanesulfonyl fluoride

GAPDH

Glyceraldehyde-3-phosphate dehydrogenase

N1ICD

Notch-1 Intracellular Domain

ANOVA

one-way analysis of variance

qRT-PCR

quantitative real-time polymerase chain reaction

ceRNA

competitive endogenous RNA

\section{Declarations}

\section{Acknowledgements}

We are grateful for the National Natural Science Foundation of China, Liaoning Provincial Higher Education Innovation Team, Distinguished Professor of Liaoning Province, China Medical University's 2018 Discipline Construction "Major Special Construction Plan", and Outstanding Scientific Fund of Shengjing Hospital.

\section{Funding}


Our study was supported by the National Natural Science Foundation of China (No. 81872123), Liaoning Provincial Higher Education Innovation Team, Distinguished Professor of Liaoning Province, China Medical University's 2018 Discipline Construction "Major Special Construction Plan” (No. 3110118029), and Outstanding Scientific Fund of Shengjing Hospital (No. 201601).

\section{Conflicts of interest}

All authors declare that they have no competing interests.

\section{Author contributions}

Yibing Li designed the study. Jianing Huo collated the clinical data of the EC patients. Junjian $\mathrm{He}$ conducted the material extraction process during the animal experiments. All authors have read and approved the final submitted manuscript.

\section{Availability of data and materials}

Not applicable.

\section{Ethics approval and consent to participate}

All patients obtained informed consent and had been approved by the Ethics Committee of Shengjing Hospital, China Medical University (2018PS251K). We conducted all animal experiments in strict accordance with a protocol approved by the Administrative Panel on Laboratory Animal Care of the Shengjing Hospital (2018PS136K).

\section{Consent for publication}

Not applicable.

\section{References}

1. Rebecca L, Siegel KD, Miller. Ahmedin Jemal. cancer statistics, 2019. CA CANCER J CLIN. 2019;69:734.

2. Koh WJ, Abu-Rustum NR, Bean S, Bradley K, Campos SM, Cho KR, et al. Uterine Neoplasms, Version 1.2018, NCCN Clinical Practice Guidelines in Oncology. J Natl Compr Canc Netw. 2018 Feb;16(2):170-99.

3. Clarke MF, Dick JE, Dirks PB, Eaves CJ, Jamieson CH, Jones DL, et al. Cancer stem cellsperspectives on current status and future directions: AACR Workshop on cancer stem cells. Cancer Res. 2006;66(19):9339-44.

4. Visvader JE, Lindeman GJ. Cancer stem cells: current status and evolving complexities. Cell Stem Cell. 2012;10(6):717-28. 
5. Huang T, Alvarez A, Hu B, Cheng SY. Noncoding RNAs in cancer and cancer stem cells. Chin J Cancer. 2013;32(11):582-93.

6. Ma Z, Wang YY, Xin HW, Wang L, Arfuso F, Dharmarajan A, et al. The expanding roles of long noncoding RNAs in the regulation of cancer T stem cells. Int J Biochem Cell Biol. 2019;108:17-20.

7. Kong FF, Li D, Yang H, Ma J, Pan X, Liu HX, et al. Preliminary identification of endometrial cancer stem cells in vitro and in vivo. Biochem Biophys Res Commun. 2017;490(2):506-13.

8. Miranda-Castro R, de-Los-Santos-Álvarez N, Lobo-Castañón MJ. Long noncoding RNAs: from genomic junk to rising stars in the early detection of cancer. Anal Bioanal Chem. 2019;411(19):4265-75.

9. Liu H, Wan J, Chu J. Long non-coding RNAs and endometrial cancer. Biomed Pharmacother. 2019;119:109396.

10. Yang B, Shen J, Xu L, Chen Y, Che X, Qu X, et al. Genome-Wide Identification of a Novel Eight-IncRNA Signature to Improve Prognostic Prediction in Head and Neck Squamous Cell Carcinoma. Front Oncol. 2019;9:898-913.

11. Ning P, Wu Z, Hu A, Li X, He J, Gong X, et al. Integrated genomic analyses of lung squamous cell carcinoma for identification of a possible competitive endogenous RNA network by means of TCGA datasets. PeerJ. 2018;6:e4254.

12. Huang $F$, Wen $C$, Zhuansun $Y$, Huang $L$, Chen $W$, Yang $X$, et al. A novel long noncoding RNA OECC promotes colorectal cancer development and is negatively regulated by miR-143-3p. Biochem Biophys Res Commun. 2018;503(4):2949-55.

13. Emmrich S, Streltsov A, Schmidt F, Thangapandi VR, Reinhardt D, Klusmann JH. LincRNAs MONC and MIR100HG act as oncogenes in acute megakaryoblastic leukemia. Mol Cancer. 2014;13:171.

14. Bartel DP. MicroRNAs: target recognition and regulatory functions. Cell. 2009;136(2):215-33.

15. Iorio MV, Croce CM. MicroRNA dysregulation in cancer: diagnostics, monitoring and therapeutics. A comprehensive review. EMBO Mol Med. 2012 Mar;4(3):143-59.

16. He Q, Huang L, Yan D, Bi J, Yang M, Huang J, et al. CircPTPRA acts as a tumor suppressor in bladder cancer by sponging miR-636 and upregulating KLF9. Aging. 2019;11(23):11314-28.

17. Jang JY, Lee YS, Jeon YK, Lee K, Jang JJ, Kim CW. ANT2 suppression by shRNA restores miR-636 expression, thereby downregulating Ras and inhibiting tumorigenesis of hepatocellular carcinoma. Exp Mol Med. 2013;45(1):e3.

18. Braun MM, Overbeek-Wager EA, Grumbo RJ. Diagnosis and Management of Endometrial Cancer. Am Fam Physician. 2016;93(6):468-74.

19. Nassar D, Blanpain C. Cancer stem cells: basic concepts and therapeutic implications. Annu Rev Pathol. 2016;11:47-76.

20. Bao B, Ahmad A, Azmi AS, Ali S, Sarkar FH. Cancer Stem Cells (CSCs) and Mechanisms of Their Regulation: Implications for Cancer Therapy. Curr Protoc Pharmacol. 2013; Chap. 14:Unit-14.25. 
21. Katoh M. Canonical and non-canonical WNT signaling in cancer stem cells and their niches: cellular heterogeneity, omics reprogramming, targeted therapy and tumor plasticity (Review). Int $\mathrm{J}$ Oncol. 2017;51(5):1357-69.

22. Coles GL, Cristea S, Webber JT, Levin RS, Moss SM, He A, et al. Unbiased Proteomic Profiling Uncovers a Targetable GNAS/PKA/PP2A Axis in Small Cell Lung Cancer Stem Cells. Cancer Cell. 2020;S1535-6108(20):30253-1.

23. Das PK, Islam F, Lam AK. The Roles of Cancer Stem Cells and Therapy Resistance in Colorectal Carcinoma. Cells. 2020;9(6):E1392.

24. Kitson SJ, Rosser M, Fischer DP, Marshall KM, Clarke RB, Crosbie EJ. Targeting Endometrial Cancer Stem Cell Activity with Metformin Is Inhibited by Patient-Derived Adipocyte-Secreted Factors. Cancers (Basel) 2019 May 11;11(5): 653.

25. Gao Y, Qian H, Tang X, Du X, Wang G, Zhang H, et al. Superparamagnetic iron oxide nanoparticlemediated expression of miR-326 inhibits human endometrial carcinoma stem cell growth. Int $J$ Nanomedicine. 2019;14:2719-31.

26. Huang X, Xiao R, Pan S, Yang X, Yuan W, Tu Z, et al. Uncovering the roles of long non- coding RNAs in cancer stem cells. J Hematol Oncol. 2017;10(1):62.

27. Deng J, Yang M, Jiang R, An N, Wang X, Liu B. Long non-coding RNA HOTAIR regulates the proliferation, self-renewal capacity, tumor formation and migration of the cancer stem-like cell (CSC) subpopulation enriched from breast cancer cells. PLoS One. 2017;12(1):e0170860.

28. Pádua Alves C, Fonseca AS, Muys BR. Brief report: The lincRNA Hotair is required for epithelial-tomesenchymal transition and stemness maintenance of cancer cell lines. Stem Cells. 2013;31(12):2827-32.

29. Wang Y, Zhu P, Luo J, Wang J, Liu Z, Wu W, et al. LncRNA HAND2-AS1 promotes liver cancer stem cell self-renewal via BMP signaling. EMBO J. 2019;38(17):e101110.

30. Wu DM, Zheng ZH, Zhang YB, Fan SH, Zhang ZF, Wang YJ, et al. Down-regulated IncRNA DLX6-AS1 inhibits tumorigenesis through STAT3 signaling pathway by suppressing CADM1 promoter methylation in liver cancer stem cells. J Exp Clin Cancer Res. 2019;38(1):237.

31. Zhou X, Gao Q, Wang J, Zhang X, Liu K, Duan Z. Linc-RNA-RoR acts as a "sponge" against mediation of the differentiation of endometrial cancer stem cells by microRNA-145. Gynecol Oncol. 2014;133(2):333-9.

32. Fang Y, Fullwood MJ. Roles, functions, and mechanisms of long non-coding RNAs in cancer. Genomics Proteomics Bioinformatics. 2016;14(1):42-54.

33. Tay Y, Rinn J, Pandolfi PP. The multilayered complexity of ceRNA crosstalk and competition. Nature. 2014;505(7483):344-52.

34. Han L, Yan Y, Zhao L, Liu Y, Lv X, Zhang L, et al. LncRNA HOTTIP facilitates the stemness of breast cancer via regulation of miR-148a-3p/WNT1 pathway. J Cell Mol Med. 2020;24(11):6242-52.

35. Xu JH, Chen RZ, Liu LY, Li XM, Wu CP, Zhou YT, et al. LncRNA ZEB2-AS1 promotes the proliferation, migration and invasion of esophageal squamous cell carcinoma cell through miR-574-3p/HMGA2 
axis. Eur Rev Med Pharmacol Sci. 2020;24(10):5391-403.

36. Kong F, Ma J, Yang H, Yang D, Wang C, Ma X. Long non-coding RNA PVT1 promotes malignancy in human endometrial carcinoma cells through negative regulation of miR-195-5p. Biochim Biophys Acta Mol Cell Res. 2018; S0167-4889(18)30169-1.

37. Pan X, Li D, Huo J, Kong F, Yang H, Ma X. LINC01016 promotes the malignant phenotype of endometrial cancer cells by regulating the miR-302a-3p/miR-3130-3p/NFYA/SATB1 axis. Cell Death Dis. $2018 ; 9(3): 303$.

38. Espinoza I, Miele L. Notch inhibitors for cancer treatment. Pharmacol Ther. 2013;139(2):95-110.

39. Yang Y, Li X, Wang T, Guo Q, Xi T, Zheng L. Emerging agents that target signaling pathways in cancer stem cells. J Hematol Oncol. 2020;13(1):60.

40. Hossain F, Sorrentino C, Ucar DA, Peng Y, Matossian M, Wyczechowska D, et al. Notch Signaling Regulates Mitochondrial Metabolism and NF-KB Activity in Triple-Negative Breast Cancer Cells via IKKa-Dependent Non-canonical Pathways. Front Oncol. 2018;8:575.

41. JPThiery $\mathrm{H}$, Acloque RYJ, Huang MANieto. Epithelial-mesenchymal transitions in development and disease. Cell. 2009;139(5):871-90.

42. Ribatti D, Tamma R, Annese T. Epithelial-Mesenchymal Transition in Cancer: A Historical Overview. Transl Oncol. 2020 Jun;13(6):100773.

43. Gao Y, Luo X, Zhang J. LincRNA-ROR is activated by H3K27 acetylation and induces EMT in retinoblastoma by acting as a sponge of miR-32 to activate the Notch signaling pathway. Cancer Gene Ther. 2020.

44. Wang Z, Li Y, Kong D, Banerjee S, Ahmad A, Azmi AS, et al. Acquisition of epithelial-mesenchymal transition phenotype of gemcitabine-resistant pancreatic cancer cells is linked with activation of the Notch signaling pathway. Cancer Res. 2009;69(6):2400-7.

45. Shao S, Zhao X, Zhang X, Luo M, Zuo X, Huang S, et al. Notch1 signaling regulates the epithelialmesenchymal transition and invasion of breast cancer in a Slug-dependent manner. Mol Cancer. 2015;14(1):28.

46. Natsuizaka M, Whelan KA, Kagawa S, Tanaka K, Giroux V, Chandramouleeswaran PM, et al. Interplay between Notch1 and Notch3 promotes EMT and tumor initiation in squamous cell carcinoma. Nat Commun. 2017;8(1):1758.

47. Wang Y, Zhong Y, Hou T, Liao J, Zhang C, Sun C, et al. PM2.5 induces EMT and promotes CSC properties by activating Notch pathway in vivo and vitro. Ecotoxicol Environ Saf. 2019;178:159-67.

\section{Supplementary Files}

This is a list of supplementary files associated with this preprint. Click to download.

- TableS2.docx

- TableS1.docx 
- FigureS1.jpg

Page 20/20 